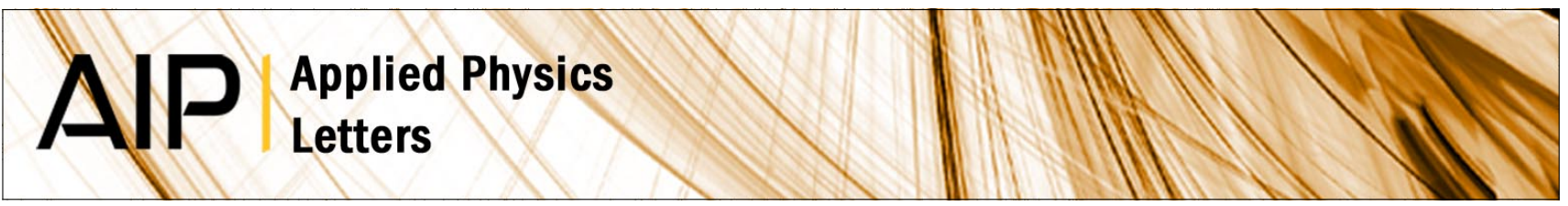

\title{
Proposal of terahertz patch antenna fed by intrinsic Josephson junctions
}

Hidehiro Asai, Masashi Tachiki, and Kazuo Kadowaki

Citation: Appl. Phys. Lett. 101, 112602 (2012); doi: 10.1063/1.4751846

View online: http://dx.doi.org/10.1063/1.4751846

View Table of Contents: http://apl.aip.org/resource/1/APPLAB/v101/i11

Published by the American Institute of Physics.

\section{Related Articles}

Reconfigurable terahertz plasmonic antenna concept using a graphene stack Appl. Phys. Lett. 101, 214102 (2012)

The analytical basis for the resonances and anti-resonances of loop antennas and meta-material ring resonators J. Appl. Phys. 112, 094911 (2012)

A metamaterial antenna with frequency-scanning omnidirectional radiation patterns Appl. Phys. Lett. 101, 173501 (2012)

A leaky-wave groove antenna at optical frequency

J. Appl. Phys. 112, 074320 (2012)

An alternative method for the measurement of the microwave temperature coefficient of resonant frequency (Tf) J. Appl. Phys. 112, 074106 (2012)

\section{Additional information on Appl. Phys. Lett.}

\section{Journal Homepage: http://apl.aip.org/}

Journal Information: http://apl.aip.org/about/about_the_journal

Top downloads: http://apl.aip.org/features/most_downloaded

Information for Authors: http://apl.aip.org/authors

\section{ADVERTISEMENT}

\section{AIP Applied Physics Letters}

\section{EXPLORE WHAT'S NEW IN APL}

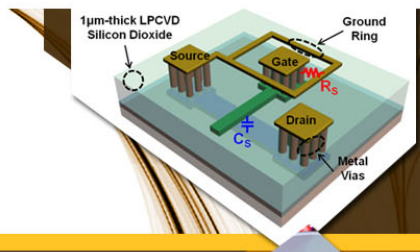

SURFACES AND INTERFACES

Focusing on physical, chemical, biological structural, optical, magnetic and electrical properties of surfaces and interfaces, and more.. 


\title{
Proposal of terahertz patch antenna fed by intrinsic Josephson junctions

\author{
Hidehiro Asai, ${ }^{1}$ Masashi Tachiki, ${ }^{2}$ and Kazuo Kadowaki ${ }^{2}$ \\ ${ }^{1}$ Electronics and Photonics Research Institute, National Institute of Advanced Industrial Science and \\ Technology (AIST), Tsukuba, Ibaraki 305-8568, Japan \\ ${ }^{2}$ Faculty of Pure and Applied Science, University of Tsukuba, Tsukuba, Ibaraki 305-8573, Japan
}

(Received 8 July 2012; accepted 27 August 2012; published online 11 September 2012)

\begin{abstract}
We propose a $\mathrm{THz}$ patch antenna, in which $\mathrm{THz}$ ac current is fed by intrinsic Josephson junctions (IJJs). The radiation power of the antenna for three different feed positions is calculated using the finite-difference time-domain method. We predict that the antenna will radiate sub-milliwatt terahertz waves with high radiation efficiency of over $20 \%$. The maximum radiation power will depend on the position of the feed. We also show that the radiation characteristics of the antenna are described well by the equivalent inductance-capacitance-resistance circuit model. (C) 2012 American Institute of Physics. [http://dx.doi.org/10.1063/1.4751846]
\end{abstract}

Over the past few decades, the search for sources of terahertz electromagnetic (EM) waves has been a hot topic in engineering fields. The terahertz EM wave has a tremendous potential in the technological applications such as non-destructive inspection, bio-sensing, and high-speed wireless communication. Various types of terahertz emitters, such as, quantum-cascade lasers ${ }^{1}$ and resonant-tunneling diodes, ${ }^{2}$ have been studied so far. However, the generation of high power EM waves in the frequency range $0.1 \sim 10$ THz, known as the "terahertz gap," is still a challenging problem.

High- $T_{\mathrm{c}}$ superconductors have received much attention as promising candidates for compact solid-state terahertz sources after Ozyuzer et al. reported the radiation of highly monochromatic, continuous terahertz wave from $\mathrm{Bi}_{2} \mathrm{Sr}_{2} \mathrm{CaCu}_{2} \mathrm{O}_{8+\delta}$ (Bi2212) single crystal. ${ }^{3}$ Cuprate high- $T_{\mathrm{c}}$ superconductors, such as Bi2212, form intrinsic Josephson junctions (IJJs), that is, natural stacks of Josephson junctions composed of the stacking of superconducting $\mathrm{CuO}_{2}$ layers and insulating layers. ${ }^{4}$ Thus, the ac Josephson current flows through the crystal under dc voltage along the $c$ axis, and radiation of EM waves is triggered by this ac current. In previous experimental studies, intense terahertz radiation has been observed for mesa-structured IJJ samples. ${ }^{3,5-10}$ The emission characteristics of the IJJ mesa have also been investigated theoretically, ${ }^{11-21}$ and these theoretical studies attribute the intense emission to the excitation of the transverse Josephson plasma. In the IJJ mesas, the transverse Josephson plasma is excited strongly under dc voltages when the ac Josephson frequencies coincide with the cavity resonant frequencies that are determined by the mesa geometries. At present, IJJ-based terahertz emitters are able to cover the frequency range of $0.3 \sim 1 \mathrm{THz}$ with the radiation power of approximately $10 \mu \mathrm{W}$.

In the IJJ mesas, large Joule dissipation occurs under dc voltages. In a typical IJJ mesa studied by Minami et al. ${ }^{5}$ for example, Joule dissipation of over $10 \mathrm{~mW}$ occurs when the mesa emits terahertz EM waves. This large dissipation implies that the radiation efficiency is extremely low, and is of order $0.1 \%$. An improvement of the radiation efficiency is a crucial task for the practical application of the IJJ-based terahertz emitter.
In this letter, we propose an IJJ-based terahertz emitter that overcomes the problem of low radiation efficiency. We perform a numerical simulation of $\mathrm{THz}$ radiation from prototype emitters and predict intense emission of sub-THz EM waves with radiation efficiency that reaches $23 \%$.

In Fig. 1(a), we show a schematic view of the terahertz patch antenna. A square metal patch is placed on a dielectric substrate, and the patch and the ground plane are electrically connected by a rectangular solid of IJJs. The dc external current injected into the metal patch flows through the IJJs to the ground plane. The IJJ layers stack along the $z$ axis, and thus the ac Josephson current flows between the patch and the ground plane when the IJJs are in resistive states. Therefore, the IJJs act as the "feed line" of the antenna.

The dimensions of the antenna in the $x-y$ plane are given in Fig. 1(b). We set the thickness of the antenna to $4 \mu \mathrm{m}$ and the relative dielectric constant of the dielectric substrate to $\epsilon_{s}=2.25$.

Of particular note is that the dimensions of the IJJ sample in our antenna are substantially small compared to those of the terahertz IJJ emitters reported in the previous studies. The dissipation in the IJJs is greatly suppressed in this antenna because of this. In previous studies, the terahertz emission was achieved by the excitation of transverse Josephson plasma waves in mesa-structured IJJs. The dimensions of these mesas are in the range $50 \sim 500 \mu \mathrm{m}$, and are comparable to the wavelength of the Josephson plasma wave. In contrast, in our antenna, the IJJs are used as the "feed" of the terahertz patch antenna, and thus the dimensions of the IJJs can be much smaller than the wavelength of the plasma wave.

We use the finite-difference time-domain (FDTD) method to investigate the time evolution of the electromagnetic field around the patch antenna and the radiation power. In this calculation, we assume that the dielectric substrate and the ground plane have infinite size in the $x-y$ plane. Moreover, we treat the metal patch and the ground plane as perfect electric conductors. In the regions outside the IJJs, we solve the three-dimensional Maxwell's equations. Inside the IJJs, we solve the dynamical equation of the phase difference and the electric field for each layer which are described as follows: 


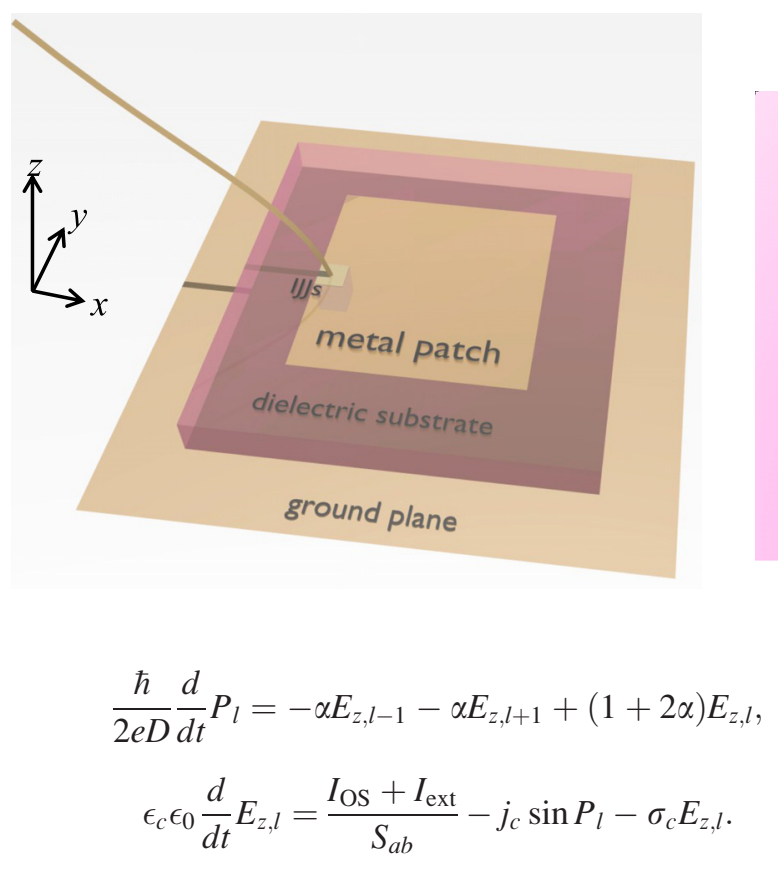

Here, $E_{z, l}$ and $P_{l}$ are the $z$ component of the electric field and the phase difference between the $l$ th and $l-1$ th layers, $\alpha$ is the capacitive coupling parameter between IJJ layers, ${ }^{22}$ $\epsilon_{c}$ is the dielectric constant of IJJs along the $c$ axis, $I_{\text {ext }}$ is the external dc current, $\sigma_{c}$ is the conductivity for the quasi particle current, $S_{a b}$ is the area of the IJJ plane parallel to the $a$ - $b$ plane, and $j_{c}=\phi_{0} /\left(2 \pi \mu_{0} \lambda_{c}^{2} D\right)$ is the critical current density of the IJJs. $D$ is the thickness of the insulating layer, $\lambda_{c}$ is the penetration depth along the $c$ axis, and $\phi_{0}$ is the flux quantum. The external oscillating current $I_{\mathrm{OS}}$ which is induced by the external field is given by $I_{\mathrm{OS}}=\int_{C} \mathrm{H}_{\mathrm{OS}} d 1$, where $C$ is the closed loop around the surface of the IJJs, $H_{\mathrm{OS}}$ is the oscillat- ing part of magnetic field. Since the electrodes are well conductive, the electric field in the electrode region $E_{z, 0}$ and $E_{z, N}$ is negligibly small compared with that in the IJJs $E_{z, 1} \sim E_{z, N-1}$. Hence, we take $E_{z, 0}=E_{z, N}=0$. In this letter, we take $\sigma=5 \mathrm{~S} / \mathrm{m}, \lambda_{c}=100 \mu \mathrm{m}, \alpha=0.1, D=12 \AA$ and the thickness of the superconducting layer $s=3 \AA$. Since the height of the IJJs is $4 \mu \mathrm{m}$ in our model, the number of IJJ layers $N$ is 2667. These values are comparable to those of Bi2212 samples. In the above equations, we assume that the $P_{l}$ and the current distribution are uniform in the $a b$ plane because the sizes of the IJJs in the $a-b$ plane are much smaller than the penetration depth $\lambda_{c}$. We consider three different positions of the IJJs $L_{\text {feed }}=20,44$, and $68 \mu \mathrm{m}\left(L_{\text {feed }}\right.$ is shown in Fig. 1$)$, and calculate the radiation power for each of these positions.

Figures 2(a) and 2(b) show the current and the radiation power as functions of voltage per IJJ layer (i.e., the total voltage divided by $\mathrm{N}$ ) for the three feed positions. In this calculation, we decrease the external current from 0.48 to
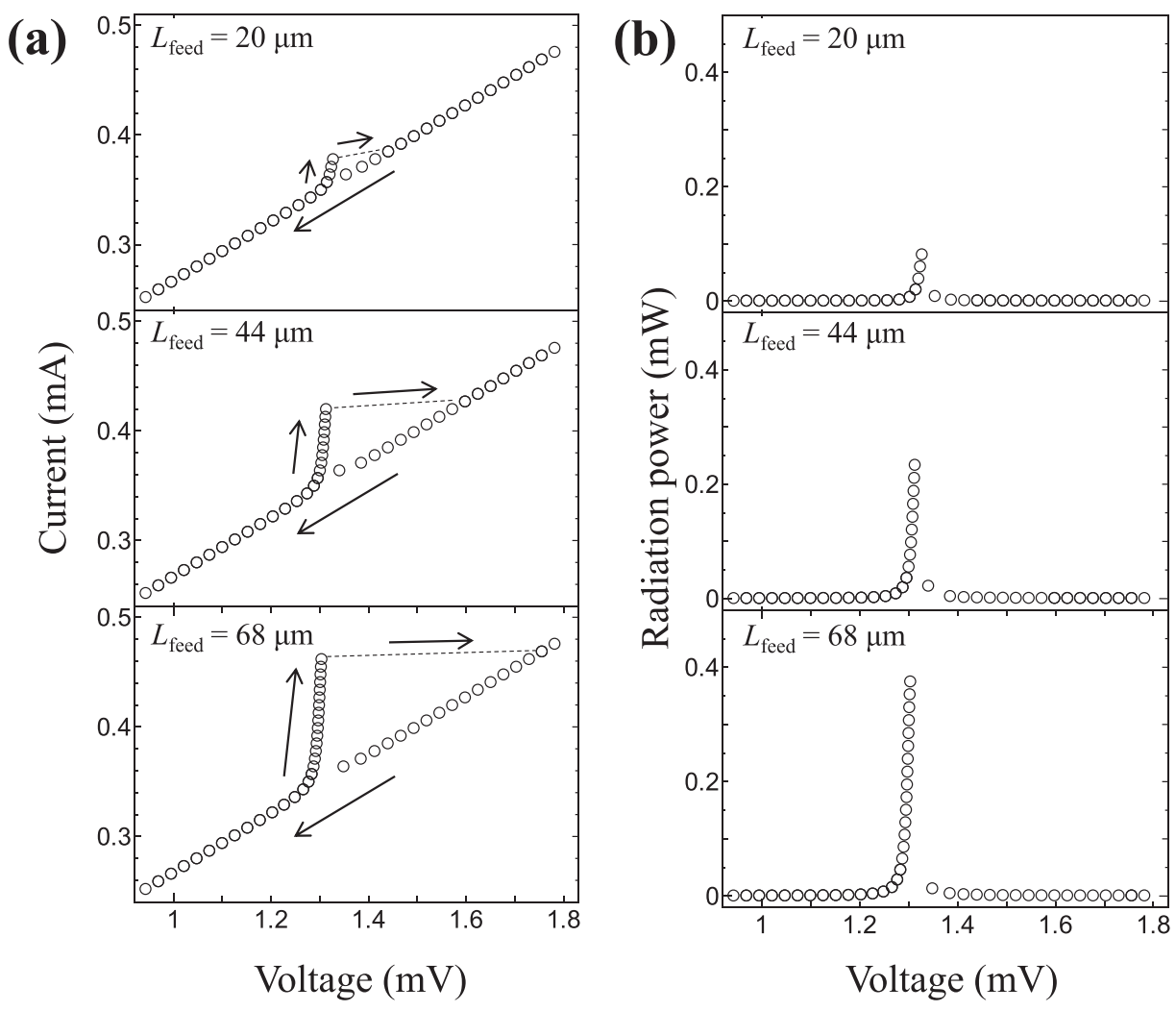

FIG. 2. (a) The current-versus-voltage $(I-V)$ curves and, (b) the radiation powerversus-voltage $(P-V)$ curves for $L_{\text {feed }}=20,44,68 \mu \mathrm{m}$. 


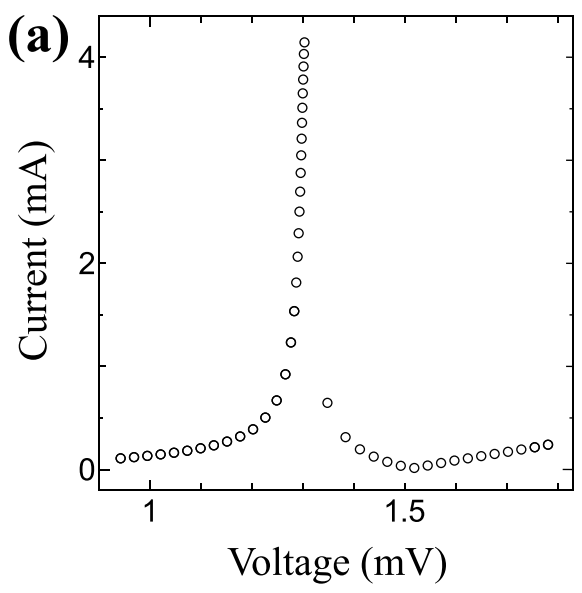

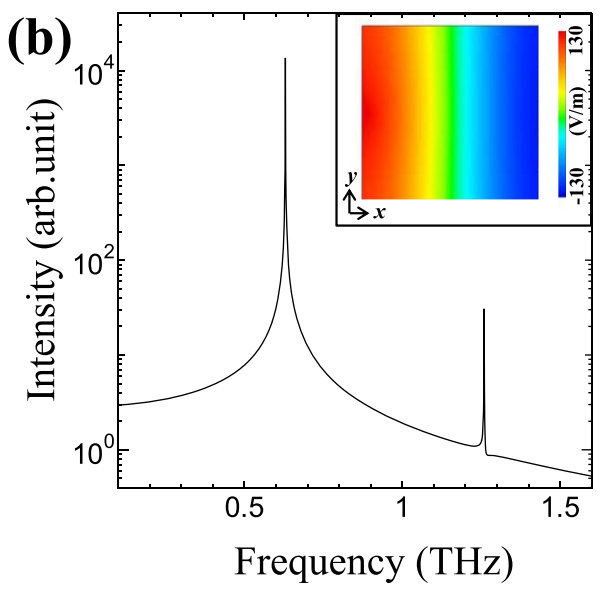

FIG. 3. (a) The displacement current as a function of voltage. (b) The frequency spectrum of the EM wave emitted by the antenna at $1.31 \mathrm{mV}$. An inset shows a snapshot of the oscillating part of $E_{z}$ at $1.31 \mathrm{mV}$.
$0.26 \mathrm{~mA}$ in the resistive state, and then increase the current from $0.26 \mathrm{~mA}$ to $0.48 \mathrm{~mA}$.

In the current versus voltage $(I-V)$ curve, a voltage step appears at $1.31 \mathrm{mV}$ for all feed positions (see Fig. 2(a)). The radiation powers sharply increase around these steps, as shown in the power versus voltage $(P-V)$ curve (see Fig. $2(b))$. The width of the steps and the radiation powers become larger as $L_{\text {feed }}$ is increased, and the maximum radiation power of $0.37 \mathrm{~mW}$ is obtained when $L_{\text {feed }}=68 \mu \mathrm{m}$ and $V=1.31 \mathrm{mV}$. Since the input power corresponding to this radiation is estimated to be $1.61 \mathrm{~mW}$ from the $I-V$ curve, the radiation efficiency is $23 \%$.

Figure 3 shows the amplitude of the displacement current in the IJJs as a function of the voltage for $L=68 \mu \mathrm{m}$. The displacement current in the IJJs is strongly enhanced at $1.31 \mathrm{mV}$. In Fig. 3(b), we show the Fourier transform of the EM wave emitted from the antenna at $V=1.31 \mathrm{mV}$ for $L_{\text {feed }}=68 \mu \mathrm{m}$. The peak frequency $0.63 \mathrm{THz}$ is exactly equal to the ac Josephson frequency $f_{J}=2 \mathrm{eV} / \mathrm{h}$. Moreover, higher harmonics $(1.26 \mathrm{THz})$ appear due to the nonlinearity of the ac Josephson effect.

The inset in Fig. 3(b) shows a snapshot of the oscillating part of $E_{z}$ at $V=1.31 \mathrm{mV}$, and this corresponds to the standing wave mode. The appearance here of the standing wave mode is due to the fact that the ac Josephson frequency $0.63 \mathrm{THz}$ is equal to the resonance frequency $f_{\mathrm{c}}$ of the funda- mental cavity mode, where $f_{\mathrm{c}}$ is given by $f_{c}=c_{0} /(2 w \sqrt{\epsilon})$, $c_{0}$ is the light velocity in free space, and $w$ is the width of the metal patch.

In this case, the field oscillation in the IJJs is amplified by receiving a strong feedback from the EM standing wave. Consequently, the radiation power of the patch antenna increases drastically. In this manner, the strong radiation peaks appear due to the commensuration between the ac Josephson frequency of the IJJs and the cavity resonance frequency of the antenna structure. Hence, the resonance voltage and the radiation frequency are uniquely determined by the geometry of the antenna. The strength of the coupling between the field oscillation inside the IJJs and the EM standing wave in the antenna depends on the position of the IJJ feed. As shown in Figs. 2(a) and 2(b), this coupling become stronger when the IJJ feed is located near the edge region of the patch.

Finally, we show that the radiation characteristics of the above patch antenna are well described by the equivalent inductance-capacitance-resistance (LCR) circuit model. Figure 4(a) shows a schematic figure of the IJJs shunted by the LCR circuit that corresponds to the patch antenna structure. In this equivalent circuit, $Q^{2} / 2 C+(L / 2)\left(d Q^{2} / d t^{2}\right)$ is the electromagnetic energy accumulated in the patch antenna. The radiation power of the antenna is expressed as the enegy loss $R I^{2}$ of the circuit. The parameters $L, C$, and $R$
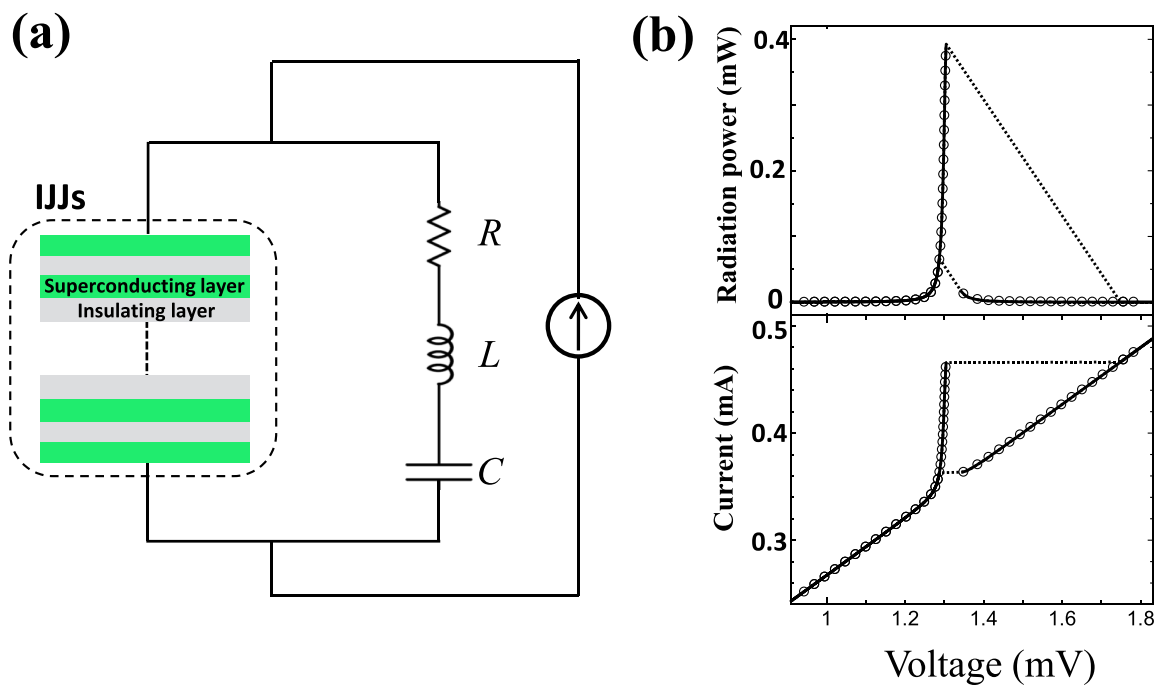

FIG. 4. (a) A schematic diagram of the equivalent LCR circuit model. (b) $I-V$ and $P-V$ curves (solid curves) calculated using the LCR circuit model. 
are the equivalent inductance, capacitance, and radiation resistance of the patch antenna, respectively. The time evolution of the charge accumulated on the equivalent capacitor $Q$ is given by

$$
L \frac{d^{2} Q}{d t^{2}}+R \frac{d Q}{d t}+\frac{Q}{C}=\sum_{l=1}^{N} \frac{\hbar}{2 e} \frac{d P_{l}}{d t} .
$$

In this circuit model, the external oscillating current density $I_{\mathrm{OS}}$ in Eq. (2) is given by $-d Q / d t$.

Based on this equivalent circuit model, we numerically evaluate the radiation power $R I^{2}$ of the antenna. In Fig. 4(b), we show the $I-V$ and $P-V$ curves (solid curves) which are obtained by solving Eqs. (1), (2), and (3) with $L=0.386 \mathrm{nH}$, $C=0.175 \mathrm{fF}, R=17.9 \Omega$. The open circles indicate the calculated values in the above FDTD simulation for $L_{\text {feed }}=68 \mu \mathrm{m}$. As seen in Fig. 4(b), the results obtained by the FDTD simulation are in good agreement with those of the LCR circuit model. The above results imply that the radiation characteristics of the patch antenna fed by IJJs are described well by the equivalent LCR circuit model, wherein the sharp peaks in the the $P-V$ curve of the antenna are attributed to the $\mathrm{LC}$ resonance.

In summary, we proposed a $\mathrm{THz}$ patch antenna fed by IJJs, and investigated the radiation characteristics of the antenna. We predicted that the antenna emits sub-milliwatt terahertz waves with high radiation efficiency over $20 \%$. We also showed that the radiation characteristics of the antenna are well described by the equivalent LCR circuit model.

We wish to thank Dr. T. Kashiwagi, Dr. H. Minami, and Mr. M. Tsujimoto (University of Tsukuba) for valuable discussions and comments. This work was supported in part by CREST-JST (Japan Science and Technology Agency).
${ }^{1}$ R. Kohler, A. Tredicucci, F. Beltram, H. E. Beere, E. H. Linfield, A. Davies, D. Ritchie, R. C. Lotti, and F. Rossi, Nature 417, 156 (2002).

${ }^{2}$ N. Orihashi, S. Suzuki, and M. Asada, Appl. Phys. Lett. 87, 233501 (2005).

${ }^{3}$ L. Ozyuzer, A. E. Koshelev, C. Kurter, N. Gopalsami, Q. Li, M. Tachiki, K. Kadowaki, T. Yamamoto, H. Minami, H. Yamaguchi, T. Tachiki, K. E. Gray, W. K. Kwok, and U. Welp, Science 318, 1291 (2007).

${ }^{4}$ R. Kleiner, F. Steinmeyer, G. Kunkel, and P. Muller, Phys. Rev. Lett. 68, 2394 (1992).

${ }^{5}$ H. Minami, I. Kakeya, H. Yamaguchi, T. Yamamoto, and K. Kadowaki, Appl. Phys. Lett. 95, 232511 (2009).

${ }^{6}$ K. Kadowaki, M. Tsujimoto, K. Yamaki, T. Yamamoto, T. Kashiwagi, H. Minami, M. Tachiki, and R. Klemm, J. Phys. Soc. Jpn. 79, 023703 (2010).

${ }^{7}$ M. Tsujimoto, K. Yamaki, K. Deguchi, T. Yamamoto, T. Kashiwagi, H. Minami, M. Tachiki, K. Kadowaki, and R. A. Klemm, Phys. Rev. Lett. 105, 037005 (2010).

${ }^{8}$ T. Kashiwagi, K. Yamaki, M. Tsujimoto, K. Deguchi, N. Orita, T. Koike, R. Nakayama, H. Minami, T. Yamamoto, R. A. Klemm, M. Tachiki, and K. Kadowaki, J. Phys. Soc. Jpn. 80, 094709 (2011).

${ }^{9}$ T. M. Benseman, A. E. Koshelev, K. E. Gray, W.-K. Kwok, U. Welp, K. Kadowaki, M. Tachiki, and T. Yamamoto, Phys. Rev. B 84, 064523 (2011).

${ }^{10}$ H. B. Wang, S. Guenon, B. Gross, J. Yuan, Z. G. Jiang, Y. Y. Zhong, M. Gruenzweig, A. Iishi, P. H. Wu, T. Hatano, D. Koelle, and R. Kleiner, Phys. Rev. Lett. 105, 057002 (2010).

${ }^{11}$ S. Lin and X. Hu, Phys. Rev. Lett. 100, 247006 (2008).

${ }^{12}$ X. Hu and S. Lin, Phys. Rev. B 78, 134510 (2008).

${ }^{13}$ L. N. Bulaevskii and A. E. Koshelev, Phys. Rev. Lett. 99, 057002 (2007).

${ }^{14}$ A. E. Koshelev and L. N. Bulaevskii, Phys. Rev. B 77, 014530 (2008).

${ }^{15}$ A. E. Koshelev, Phys. Rev. B 78, 174509 (2008).

${ }^{16}$ T. Koyama, H. Matsumoto, M. Machida, and K. Kadowaki, Phys. Rev. B 79, 104522 (2009).

${ }^{17}$ T. Koyama, H. Matsumoto, M. Machida, and Y. Ota, Supercond. Sci. Technol. 24, 085007 (2011).

${ }^{18}$ R. A. Klemm and K. Kadowaki, J. Phys. Condens. Matter 22, 375701 (2010).

${ }^{19}$ M. Tachiki, S. Fukuya, and T. Koyama, Phys. Rev. Lett. 102, 127002 (2009).

${ }^{20}$ M. Tachiki, K. Ivanovic, K. Kadowaki, and T. Koyama, Phys. Rev. B 83, 014508 (2011).

${ }^{21}$ H. Asai, M. Tachiki, and K. Kadowaki, Phys. Rev. B 85, 064521 (2012).

${ }^{22}$ T. Koyama and M. Tachiki, Phy. Rev. B 54, 16183 (1996). 